\title{
ASTROMETRY WITH THE HST FINE GUIDANCE SENSORS
}

\author{
M. G. Lattanzi, ${ }^{1,2}$ B. Bucciarelli, ${ }^{2}$ S. T. Holfeltz, and L. G. Taff \\ Space Telescope Science Institute \\ 3700 San Martin Drive \\ Baltimore, MD 21218 USA
}

ABSTRACT. This paper presents an overview of the current status of astrometry with the Fine Guidance Sensors (FGSs) on the Hubble Space Telescope. The FGSs have two astrometric modes of operation, one for positional (POS) and one for transfer function (TRANS) astrometry. The positional mode is intended for parallax and angular velocity work. Owing to optical aberrations in the Optical Telescope Assembly (OTA) (i.e., the main telescope optics) beyond the spherical aberration, and instabilities within the combined OTA/FGS optical system, it is undergoing additional feasibility review. Preliminary engineering tests showed changes in stellar pair positions of $\sim 100$ milli-arc seconds which are far too large to allow for the expected $\sim 1$ mas calibration. Alternate calibration procedures and data reduction algorithms are currently being developed to maximize the scientific potential of this mode which can be fulfilled at the $\sim 3$ mas level.

The other operational mode-primarily intended for work on double stars but also usable for angular diameter determination - has proven more stable. Even the deleterious effects of the thermally induced solar array jitter have mostly been overcome. A large collection of reduction and analysis software is available to support this measurement technique (Lattanzi, Bucciarelli, Holfeltz, and Taff 1992) and its calibration is proceeding apace. Interesting scientific work from the FGSs on bright Hyades binaries and the highly eccentric double star ADS 11300 has already been published (Franz et al. 1991, 1992).

Finally, a new FGS Instrument Handbook (Taff 1992a) has been prepared which should minimize the complications of dealing with the General Observer Proposal Forms.

\section{OVERVIEW}

This paper first presents a brief overview of the Fine Guidance Sensor (FGS) electro-optical system and the two modes of operation of most relevance to astrometry. The third section very briefly summarizes the results of the Orbital Verfication tests for the astrometer FGS, the one in radial bay number 3 . The fourth section briefly reprises some scientific results already obtained. An Appendix addresses 0.1 milli-arc second data reduction.

\footnotetext{
${ }^{1}$ Affiliated with the Astrophysics Division, Space Science Department, ESA.

${ }^{2}$ On leave from Torino Observatory.
} 


\section{FINE GUIDANCE SENSOR OPERATION}

The Fine Guidance Sensors (FGSs) of the Hubble Space Telescope serve two functions. Their primary role is to control the high precision pointing of the spacecraft. The same optical system, detectors, and control electronics can also be utilized, if properly calibrated (Taff 1990), to perform (the secondary role of) astrometric observations of single stars, of binary systems, and to indirectly measure other properties such as angular size (Taff 1991).

The positional measurement precision demands for the FGSs are at the milli-arc second (mas) level. This is necessary to yield positions, which over the course of time, will yield correspondingly good parallaxes and proper motions. For binary star separations a precision of a few mas, for separations in the $>10$ mas range, is achievable. The errors in the position angle are expected to be $\pm 0{ }^{\circ} 1$ while those in the magnitude difference will be at least $\pm 0^{m} .2$. This performance rapidly degrades with increasing magnitude difference, especially beyond $\Delta m=3^{m}$, and decreasing separation between the components (Lattanzi et al. 1992). The total magnitude of the binary, and thence magnitudes for each component, comes from an independent $\pm 1 \%$ photometric calibration of the FGS detectors.

A new role for the FGSs is apparent size determination. We anticipate a precision of a few mas for diameters in the 15-50 mas range. Note that sizes are indirectly deduced from effects in the Transfer Function. Non-point source and binary star astrometry all rely on the departures of the Transfer Function from its nominal form. The Transfer Function is the name for the fringe visibility curve produced by the Koester's prism interferometer in the FGS units.

There are three FGSs. Each FGS is composed of an optical system, a detector system consisting of four photomultiplier tubes, and a control computer. The optical train of an FGS is shown in Bradley et al. (1991). Light converging toward the focal plane of the Optical Telescope Assembly (i.e., the main optics or OTA) is deflected by a plane pick-off mirror. This mirror's shape is approximately a quarter annulus. From there the light is eventually reimaged at each field stop of each of the four photomultiplier tubes. Prior to this the light was split into two equal beams, each beam was linearly polarized, and separately passed through two orthogonal Koester's prism interferometers. Each prism has two exit beams, each of which is focussed by a positive doublet onto the photomultiplier tube field stop assembly. This field stop limits the area actively sampled by the FGS detectors to $5^{\prime \prime} \times 5^{\prime \prime}$.

There are two operating modes of the FGS pertinent to astrometry. In one mode, and in Fine Lock guidance, the interference function is used to acquire and then lock-on to the light source. This Transfer Function (TF) is the normalized difference between the signals of the two photomultiplier tubes per axis. Because of the $50-50 \%$ intensity splitting of the polarizing beam splitter, there is sensitivity in two orthogonal directions. Hence we speak of the $x$ and $y$ axes within an FGS and there are two TFs for each FGS. Fine Lock occurs when each axis is independently maintained at its null position. If we denote the counts, on (say) the $y$ axis, as $A_{y}$ and $B_{y}$ from the two output channels of the prism, then the $y$ axis TF $\left(S_{y}\right)$ is given by $S_{y}=\left(A_{y}-B_{y}\right) /\left(A_{y}+B_{y}\right)$. The pre-launch expectation for a perfect optical system and a point source, monochromatic, single star TF was $S(z)=\sin ^{2} z / z(z=2 \pi R \theta / \lambda$ where $R$ is the radius of the primary mirror of the OTA, $\lambda$ is the wavelength, and $\theta$ is the angle from the point of perfect interference). A real TF is shown in Fig. 1.

When an FGS has locked on to a star, that is, found the null in each of its two TFs, this condition is maintained via a servo feedback loop. Once this state is maintained in a stable fashion the direction to the star can be determined. Repeating this lock-on process with several nearby reference stars completes the relative positional determination.

For non-positional astrometry the $5^{\prime \prime}$ square instantaneous field-of-view of the FGS is driven across the star. The step size (as small as 0.3 mas) can be controlled via telemetry. As the interferometer null is approached and passed the TF reacts in a characteristic fashion to the program object. The deformation of an observed $\mathrm{TF}$ from the reference TF obtained from a single, point source contains the information we want (see Taff 1991 for examples). 


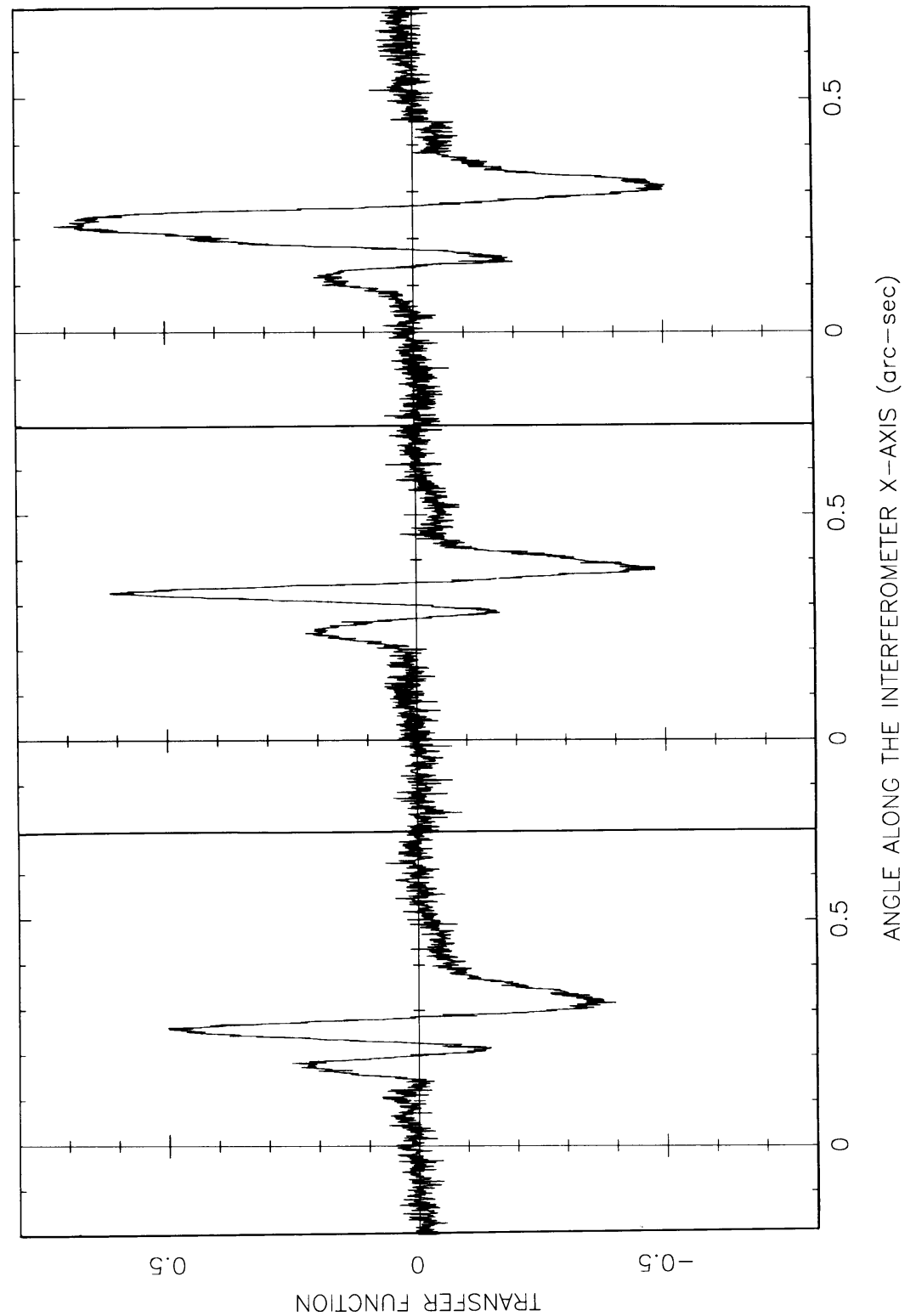

量 


\section{SUMMARY OF SPACECRAFT TESTS}

The Orbital Verification phase for HST FGS Astrometry was designed to test the engineering aspects of the FGSs. The original Orbital Verification (OV) plan consisted of many tests of each of the three FGS units to determine which was best suited for scientific work. The discovery of spherical aberration in the primary mirror (Burrows et al. 1991) and the subsequent discovery of internal misalignments within each FGS (Benedict et al. 1991) have resulted in many delays to the HST FGS astrometric program. Much effort, especially by the Space Telescope Astrometry Team, has been devoted to finding a secondary mirror position which would result in at least one FGS being usable as an astrometer. The final HST collimation involved some compromise.

Results from two OV tests are reported below. These include Astrometry Verification and a Nine Points of Light test (see Benedict et al. 1991 for a test description). The stars successfully observed are listed in Table 1 . It includes the name, the $V$ magnitude, the filter, the measured $x, y$ position and their associated standard deviations, and the Fine Error Signal averaging duration. The stars are all located near NGC 188. The fainter stars are from Sandage (1962); the brighter stars are listed in Upgren, Mesrobian, and Kerridge (1972).

\subsection{POS Mode}

The POS mode observations in Table 1 are grouped by orbit starting on day 359 of 1991 . The positional differences between orbits are immaterial. (Drifts of $\sim 1^{\prime \prime}$ are to be expected between successive Guide Star acquisitions.) Positional differences within an orbit are an indication of the POS mode measurement precision and are generally the same size as $\sigma_{x}$ and $\sigma_{y}$.

The F583W (or CLEAR filter) limiting magnitude and positional precisions presented below for FGS 3 should be considered the best possible. For the same stars observed through the same filter, FGS 3 appears to be 0.28 mag more sensitive than FGS 1 .

If we define successful astrometry as having internal errors of position of less than 0.003 arcsec (standard deviation), from Table 1 we see that the F583W limiting magnitude in FGS 3 is $V=17^{m}$. One can astrometrically detect Sandage-I118 at the center of the field-of-view. Given the TF variations within FGS 3, one is not likely to reach $V=17^{m}$ everywhere (see below). Finally, note too that $\sigma_{y}>\sigma_{x}$. Spacecraft jitter is greater along the FGS $3 y$ axis because this axis is co-linear with the solar array rotation axis.

Averaging over all measurements, $\left\langle\sigma_{x}\right\rangle=2.6$ and $\left\langle\sigma_{y}\right\rangle=2.9$ mas. It is apparent that, for the same signal-to-noise ratio, particularly for the fainter stars, the measurement errors are less for PUPIL observations than for any full-aperture observations of the same star. The reason that astrometry with the PUPIL in place is generally better than with any of the other filters is simple to understand once one remembers that the PUPIL filter is essentially an aperture stop. It prevents light from the outer annulus of the telescope's focal plane from entering the FGS. Since this outermost $1 / 3$ is where the spherical aberration of the the primary mirror has most distorted the initial phase coherence of the incoming wave front from the star, the fringe visibility function of the Koester's prism, and hence everything that depends on it, is much enhanced with the PUPIL in place.

It is also possible to perform on-board averaging of the "Fine Error Signal." This should provide higher quality data, especially for fainter stars. Comparing lines 12 and 14 and lines 8 and 13 in Table 1, we see that FES averaging does generally have the expected effect. The standard deviations about the mean do drop with increasing exposure time.

\subsection{TRANS Mode}

Fig. 1 and Fig. 2 for FGS 3 show the end result of many months of effort by the HST Project, the ST ScI, and the ST Astrometry Team. Each TF was obtained by scanning the star Upgren-69 (Upgren, Mesrobian, and Kerridge 1972). We present the TFs obtained 


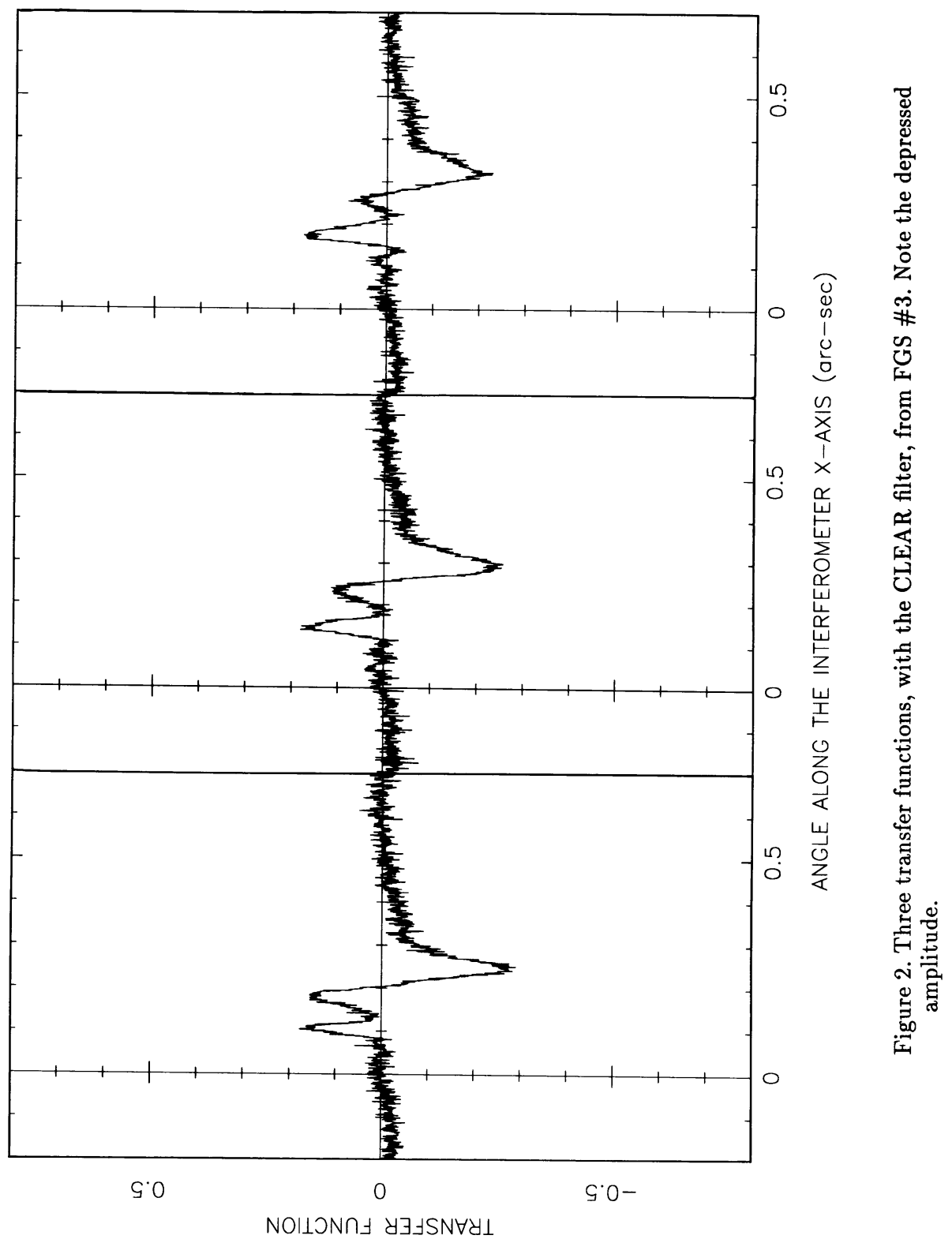


TABLE 1. FGS 3 POS Mode Results

\begin{tabular}{cccccccc}
\hline \multicolumn{1}{c}{ Name } & $V$ & Filter & $x$ & $y$ & $\sigma_{x}$ & $\sigma_{y}$ & FES Time \\
\hline Upgren-111 & $12^{m} \cdot 41$ & F583W & $5^{\prime \prime} 4325$ & $7266^{\prime \prime} 2195$ & $0^{\prime \prime} 0019$ & $0 !^{\prime \prime} 0026$ & 0.025 \\
Upgren-111 & 12.41 & F605W & 5.4379 & 726.2313 & 0.0028 & 0.0020 & 0.1 \\
Upgren-111 & 12.41 & F550W & 5.4336 & 726.2307 & 0.0019 & 0.0023 & 0.1 \\
Upgren-111 & 12.41 & PUPIL & 5.4342 & 726.2235 & 0.0017 & 0.0024 & 0.05 \\
& & & & & & & \\
Upgren-112 & 10.84 & F550W & 5.8083 & 726.8494 & 0.0019 & 0.0031 & 0.05 \\
& & & & & & & \\
Upgren-111 & 12.41 & F550W & 5.3859 & 726.2795 & 0.0019 & 0.0030 & 0.05 \\
Upgren-111 & 12.41 & F550W & 5.3864 & 726.2787 & 0.0019 & 0.0029 & 0.2 \\
& & & & & & & \\
Upgren-108 & 15.03 & F550W & 5.4020 & 726.1275 & 0.0040 & 0.0047 & 0.2 \\
& & & & & & & \\
Upgren-112 & 10.84 & PUPIL & 4.1320 & 726.8283 & 0.0022 & 0.0031 & 0.025 \\
Upgren-111 & 12.41 & PUPIL & 4.6038 & 727.0945 & 0.0018 & 0.0028 & 0.05 \\
Upgren-111 & 12.41 & PUPIL & 4.6053 & 727.0947 & 0.0021 & 0.0020 & 0.2 \\
Upgren-108 & 15.03 & PUPIL & 4.6551 & 726.9406 & 0.0029 & 0.0038 & 0.2 \\
Upgren-108 & 15.03 & F550W & 4.6585 & 726.9499 & 0.0028 & 0.0017 & 1.6 \\
Upgren-108 & 15.03 & PUPIL & 4.6556 & 726.9408 & 0.0023 & 0.0016 & 1.6 \\
Sandage-I118 & 17.02 & F583W & 4.0824 & 727.9982 & 0.0032 & 0.0058 & 3.2 \\
Sandage-I95 & 16.06 & F583W & 4.7259 & 723.0957 & 0.0024 & 0.0032 & 1.6 \\
Sandage-I95 & 16.06 & PUPIL & 4.7317 & 723.0965 & 0.0023 & 0.0028 & 3.2 \\
Sandage-I118 & 17.02 & F583W & 4.5308 & 727.2045 & 0.0033 & 0.0025 & 3.2 \\
Sandage-I95 & 16.06 & F583W & 4.0315 & 723.6614 & 0.0032 & 0.0043 & 1.6 \\
Sandage-I95 & 16.06 & PUPIL & 4.0339 & 723.7639 & 0.0057 & 0.0029 & 3.2 \\
\hline & & & & & & &
\end{tabular}

through the F583W, or CLEAR filter (Fig. 2), and the PUPIL (Fig. 1) aperture only. As noted above, use of the PUPIL significantly reduces the aberration effects, but it also lowers the limiting magnitude by $0^{m}$.75. FGS 3 has the highest quality TFs in the center of the field-of-view. This quality is not uniform over the entire field-of-view.

Franz et al. 1991 present a successful TRANS mode observation of a relatively bright binary star (primary $=8 \cdot m$ ), ADS 11300 . They resolved it with a $\Delta m=0{ }^{m} \cdot 41 \pm 0{ }^{m} 07$ and separation $=66 \pm 3$ mas. Since the modulation and even the detectability of the TF decrease as the noise increases, a major concern was the TF quality for faint stars. One can estimate a limiting magnitude, using successful F583W and partially successful PUPIL TRANS observations of Sandage-I95. We conclude that FGS 3 could provide successful TRANS mode observations for stars of $V<16^{m}$.

\section{FGS SCIENCE TO DATE}

The ST Astrometry Team has been intensively observing Proxima Centauri in an attempt to characterize the small-scale changes in the FGS optical system. Using a network of nearby 
reference stars, and performing a relative reduction of 6 sets of observations spanning only 52 days, an annual parallax and proper motion for Proxima Centauri have been deduced. The FGS value for $\pi$ is $00^{\prime \prime} 777$ vs. the ground-based value of $0^{\prime \prime} 773$ (Kamper and Wesselink 1978; Benedict 1992). The latter rests on 130 plates covering 45 years. The proper motions agree equally well, $\mu_{\alpha}=-3^{\prime \prime} \cdot 693 / \mathrm{yr}$ and $\mu_{\delta}=0{ }^{\prime \prime} 785 / \mathrm{yr}$ for the FGSs while the ground-based values from the above reference are $-33^{\prime \prime} 766 / \mathrm{yr}$ and $0.787 / \mathrm{yr}$ respectively. Since astrometric precision goes as the square of the time interval, these results are startingly good for an incompletely calibrated instrument.

Figure 3 shows the work of Franz et al. (1991) on the highly eccentric binary star ADS 11300 . Figure $3 \mathrm{~b}$ is a blowup of the periastron region with only FGS points plotted. The milli-arc second fit is clear and the refinement of the line of apsides and the time of periastron passage have been considerable. Once again the reader must remember there are only a few months of FGS data and the instrument is still incompletely calibrated. The older ground-based data will soon become superfluous.

\section{DISCUSSION AND CONCLUSIONS}

In December of 1991 HST was not a stable platform from which to carry out 3 mas precision astrometry. In the period 24-29 December 95 POS and TRANS mode observations were attempted for OV. Of these, 32 were adversely affected by jitter. Either the shaking was of high enough amplitude to kick the guiding FGS out of Fine Lock, or, even though Fine Lock was held, the astrometry FGS was not held stable, increasing the observational errors to an unacceptable level. The almost always higher $y$ axis standard deviations (compared to those for the $x$ axis) argue that nearly all these data are to some degree tainted by HST jitter. A new servo control law was placed on board HST in 1992 April. Preliminary results suggest that the response of the guiding FGS to terminator disturbances has been improved. Loss of lock is occurring less frequently. However, this is not a totally satisfactory fix. The solar arrays still flex with each sunrise and sunset and the vehicle continues to be disturbed. The upcoming servicing mission to replace the solar arrays is essential to obtain the very best astrometry that the HST is capable of providing.

In conclusion, from on-orbit performance FGS 3 has been demonstrated to have better astrometric qualities than FGS 1, prompting the ST Astrometry Team to choose FGS 3 to be the prime astrometer. Specifically, FGS 3 has been shown to produce POS mode measurements on a star with $V=17^{\mathrm{m}}$ with a per-axis precision of 3 mas. TRANS mode appears to be a viable technique at least down to $V=16^{m}$.

\section{APPENDIX: MILLI-ARC SECOND ASTROMETRIC DATA REDUCTION}

This Appendix summarizes the astrometric corrections necessary to achieve a milli-arc second level of precision. This can be viewed as a continuation of the Porter and Sadler (1953) and Scott and Hughes (1964) papers. A key simplification of the design of our computer software turns out to be astrometrically necessary at this level of precision: to wit, we process each photon integration period fully and independently of every other one. This ensures that untreated differential astrometric effects can not build up to unacceptably high levels of error through their time integration.

Although the main purpose of the POS Mode capability of the Astrometer FGS is to carry out relative astrometry, our reduction code also implements the task of transforming relative, apparent stellar positions expressed in FGS coordinates, to absolute, true positions given in the solar system barycentric equatorial reference frame equinox J2000.0. The epoch of date is defined to be the mid-point of the photon integration time. This interval is at most $3.2 \mathrm{sec}$ for the FGSs. Such a transformation will of course introduce errors; nevertheless, we want to stress that bringing the data into an absolute system does not imply spoiling in any 


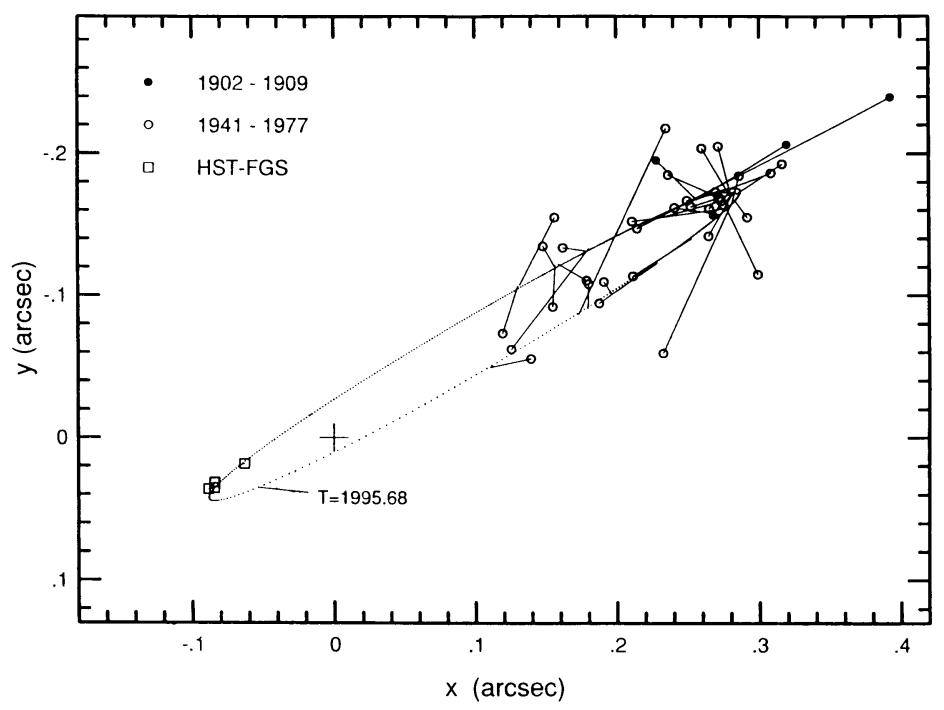

Figure 3a. All observational data for ADS 11300.

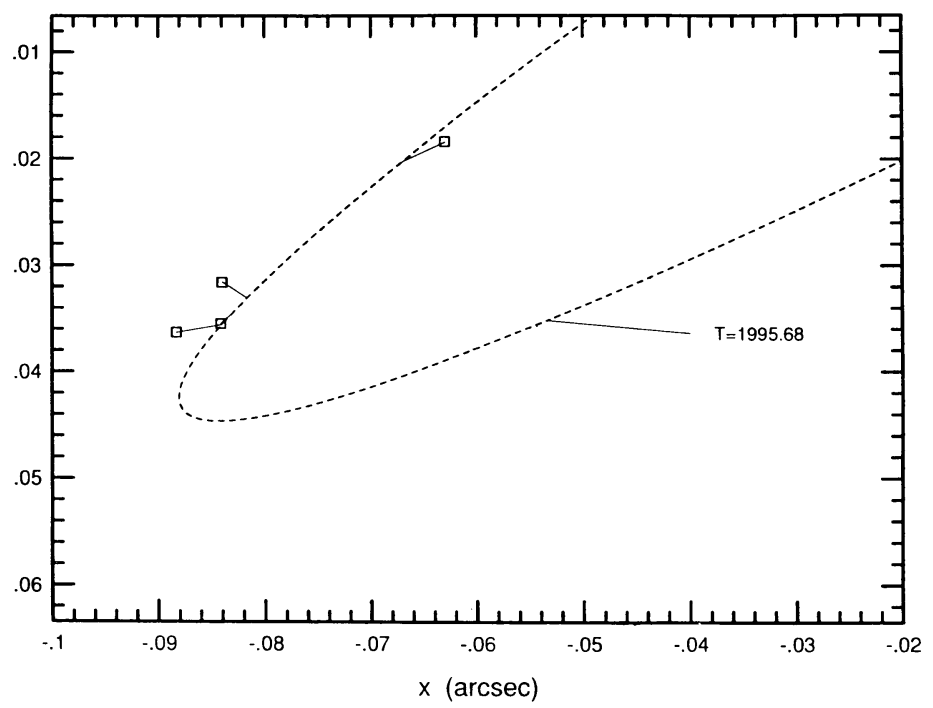

Figure 3b. HST FGS observations for ADS 11300. 
way the quality of the measurements, insofar as systematic effects which are constant on a scale of few arc minutes will be eliminated when relative positions are constructed.

The steps executed to compute absolute stellar positions are described below. First, the HST satellite location and velocity vectors need to be transformed from the Geocentric Inertial Frame (GCI) to the solar system barycentric equatorial frame. Special relativistic effects are taken into account. Next, the star's apparent position vector, which is given in an HST coordinate system--the so-called V1, V2, V3 space-is put into the equatorial system via a coordinate transformation which utilizes the spacecraft attitude data. (Note that this rests on the data for the Guide Stars given in the Guide Star Catalog and controls the accuracy of the result but not its precision (Taff et al. 1990). Also note that whatever the systematic errors in the GSC positions they will be identically transmitted to all our deduced stellar positions.) Finally, apparent place effects are removed from the star's position. This is performed in a general relativistic framework taking into account second order terms of aberrational displacement owing to the satellite's velocity, annual parallax effect, and light deflection from the Sun (the major planets Jupiter and Saturn will also be included in a subsequent version of the reduction code). A black diagram is shown in Fig. 4.

Let us define $\left(x_{e}, y_{e}, z_{e}\right)$ and $(u, v, w)=\mathbf{v}_{e}$ as the vectors representing the Earth's location and velocity in the solar system barycentric equatorial reference frame. An asterisk will indicate quantities expressed relative to the center of mass of the Earth (i.e., GCI), and a single quote mark will indicate apparent place positions. Also, define $\mathbf{R}=(X, Y, Z)$ and $\mathbf{V}$ as the HST location and velocity vectors. Finally, $\mathbf{s}$ is the solar system barycentric location vector of the target being observed.

To obtain the HST's location and velocity in the solar system barycentric frame at a generic instant of time $t^{*}$ (as given by the clock onboard the spacecraft) we use the inverse of the generalized Lorentz transformations given in Green (1988, p. 67-69), namely

$$
\begin{aligned}
& X=\left[1+\frac{\beta^{2}}{(\beta+1) c^{2}} u^{2}\right]\left(x^{*}+x_{e}\right)+\frac{\beta^{2}}{(\beta+1) c^{2}} u v\left(y^{*}+y_{e}\right)+\frac{\beta^{2}}{(\beta+1) c^{2}} u w\left(z^{*}+z_{e}\right) \\
& Y=\frac{\beta^{2}}{(\beta+1) c^{2}} u v\left(x^{*}+x_{e}\right)+\left[1+\frac{\beta^{2}}{(\beta+1) c^{2}} v^{2}\right]\left(y^{*}+y_{e}\right)+\frac{\beta^{2}}{(\beta+1) c^{2}} v \vee v\left(z^{*}+z_{e}\right) \\
& Z=\frac{\beta^{2}}{(\beta+1) c^{2}} u w\left(x^{*}+x_{e}\right)+\frac{\beta^{2}}{(\beta+1) c^{2}} v w\left(y^{*}+y_{e}\right)+\left[1+\frac{\beta^{2}}{(\beta+1) c^{2}} w^{2}\right]\left(z^{*}+z_{e}\right)
\end{aligned}
$$

for the location and

$$
\mathrm{V}=\left(\frac{\mathrm{V}^{*}}{\beta}+\mathbf{v}_{e}+\frac{\beta \mathbf{v}_{e} \cdot \mathbf{V}}{(\beta+1) c^{2}} \mathbf{v}_{e}\right) /\left(1+\frac{\mathbf{v}_{e} \cdot \mathbf{V}^{*}}{c^{2}}\right)
$$

for the velocity.

Eq. (1) does not take into account a transformation of the time coordinate. This means treating the spacecraft clock time as absolute. The final transformation from apparent place to geometric position in the solar system barycentric frame is given by the formula

$$
\mathbf{s}=\mathbf{s}^{\prime}-\left[\Pi-\frac{2 m}{R\left(R+\mathbf{R} \cdot \mathbf{s}^{\prime}\right)}\right] \mathbf{s}^{\prime} \mathbf{x}\left(\mathbf{s}^{\prime} \times \mathbf{R}\right)+\frac{1}{c}\left(1+\frac{\mathbf{V} \cdot \mathbf{s}^{\prime}}{c}\right) \mathbf{s}^{\prime} \mathbf{x}\left(\mathbf{s}^{\prime} \times \mathbf{V}\right)+\left(\frac{1}{2 c^{2}}\right) \mathbf{V} \mathbf{x}\left(\mathbf{V} \times \mathbf{s}^{\prime}\right)
$$

(see Green, p. 205), where $\Pi$ is the stellar parallax in radians, and $m=G M_{\odot} / c^{2} \sim 1.5 \mathrm{~km}$ is the light deflection constant for the solar gravitational field.

Stellar proper motion, annual parallax, and binary star orbital motion become an issue when one can perceive changes in direction as a consequence of them, during the course of an observation, at the mas level. First consider the case of an FGS Transfer Function (TF) observation on a binary star. If we wish to achieve mas measurement precision for the 
separation, then a fortiori the relative motion of the double must be less than this during the course of an observation. Let $P$ be the binary's orbital period, $a$ its semi-major axis, $r$ its distance from the Sun, and $\tau$ the individual photon integration time-of which there are $N$ necessary to form the full TF-then the constraint on the angular motion is

$$
\Delta \psi=\left(\frac{2 \pi N \tau}{P}\right) *\left(\frac{a}{r}\right)<1 \text { mas. }
$$

The derivation of this formula is straightforward. During the $N$ time intervals $\tau$ the mean anomaly of the secondary will have changed by $\Delta M=2 \pi N \tau / P$. The inscribed chord subtended by this arc is $\sim a \Delta M$ and this represents an angle $(a / r) \Delta M$ radians to an observer a distance $r$ from the binary. The minor effects of orbital eccentricity can be deduced from Lambert's theorem. Similarly, the orientation of the orbit in space must be considered. It brings in well-behaved trigonometric functions of the three Euler angles that specify it. Hence, Eq. (2) is a reliable guide.

For equality with a $0.1 \mathrm{mas}$, a TF acquisition time of $40.8 \mathrm{~min}$ (the maximum number of samples [765] for $3.2 \mathrm{sec}$ each), $P$ in hours for two solar mass stars, and the annual parallax $\Pi$ of the binary in arc seconds, Eq. (2) reads $0.00790 P^{1 / 3}=\Pi$. Hence, only for the closest and fastest binaries could this be a problem.

The issue for annual parallax is could a star with a radial velocity $v_{r}$ move far enough during the integration time for this to be detectable at 1 mas if it is a distance $r$ away (also see Stumpff 1985 and Schwarzschild 1894 and the discussion of secular parallax below)? In symbols, can any star bound to the Galaxy satisfy $v_{r} N \tau / r=0.1$ mas? Using $v_{r}=$ $400 \mathrm{~km} / \mathrm{sec}, r=1 \mathrm{pc}, \tau=3.2 \mathrm{sec}$, and $N=16$ (the maximum number of grouped integration times for the FGSs in its POSitional mode) we find that $v_{r} N \tau / r=0.14$ mas. Therefore, while the potential angular displacement of an extremely close, very fast moving star during its photon integration time is not a problem, repeated observations of the same star over many such time integrations (within a larger observing sequence that, for instance, includes similar measures on a local network of reference stars) might be. The correct treatment of this issue is to analyze the full six-dimensional kinematical state of the program object as outlined in Taff (1992b).

Finally, for the proper motion effect to be appreciable the constraint is of the form $\mu N \tau=0.1$ mas where $\mu$ is the total proper motion and $N$ is the number of photon integration times of duration $\tau$. Even at $10^{\prime \prime} / \mathrm{yr}$ and $N=16$, the largest $\mu N \tau$ can be is 0.016 mas. Including the time to observe ten reference stars with the FGSs (and therefore having to interleave additional such observations), the problem is at the 0.2 mas level.

Each of the topics just discussed has a parallel within the solar system. The analog of a double star is clearly a planetary moon revolving about its planet. The constraint is still given by a relationship of the form in Eq. (2) except that the relevant distance is now the distance to the planet. The effect will be maximized for the closest moon of the nearest planet. The solar system analogs of proper motion and parallax are to be found in the motions of comets or minor planets. The expression on the left hand side of Eq. (3) is 7 mas for an asteroid $0.2 \mathrm{~A}$. U. from the Earth moving relative to the Earth with a heliocentric escape speed of $30 \sqrt{2} \mathrm{~km} / \mathrm{sec}(N=1$ and $\tau=0.025 \mathrm{sec})$. The analogs of stellar annual parallax must be applied to the directions deduced for solar system objects so that the differential correction of their orbital elements may be successfully carried out. In practice, because of the tradition of incorporating this step within the differential correction process itself (and the non-trivial issue of photocenter vs. center of mass for finite disc objects viewed with the FGSs), we do not perform this adjustment.

Secular aberration, as for instance discussed by Schwarzschild (1894) or Stumpff (1985), plays no role in space-based observing in particular. It does play a role in the reduction of high precision astrometric data acquired over the course of time. However, as has already been alluded to, the proper method of dealing with this issue is to perform a full, physically correct, six-dimensional analysis of the data.

Because the spacecraft is in orbit about the center of mass of the Earth, there is a parallactic correction necessary to reduce the observed direction of a program object to a 


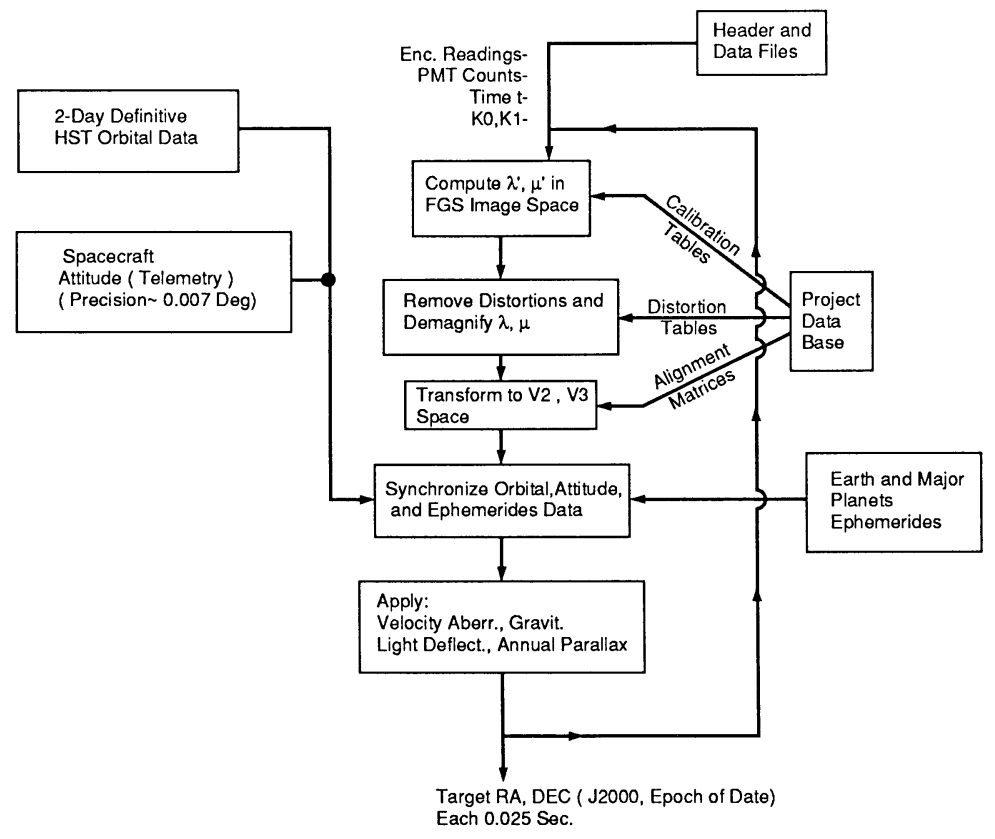

Pos Mode Code Block Diagram

Figure 4 
geocentric reference frame. For a typical main-belt asteroid this correction is not much larger than traditional diurnal parallax ( $\sim 5.7$ owing to HST's elevation of $\sim 615 \mathrm{~km}$ ). (It is in excess of 300 mas for the HST over the maximum exposure time of $51.2 \mathrm{sec}$.) These results follow from the relationship $\Delta \Pi=\Delta \psi$ where $\Delta \psi$ was given in Eq. (2). $P$ is now the HST orbital period, $a$ is its semi-major axis, and $r$ is the distance to the asteroid. The use of Lambert's theorem will provide eccentricity-dependent corrections and the products of the sines and cosines of the relative inclination, arguments of perihelion and perigee, and longitudes of the ascending nodes are required for a rigorous result. (All this trigonometry can only reduce the amplitude just derived.) Walter (1985) and Walter et al. (1986) should also be consulted.

\section{REFERENCES}

Benedict, G. F. 1992, private communication.

Benedict, G. F., Jefferys, W. H., Wang, Q., Whipple, A. L., Nelan, E., Story, D., Duncombe, R. L., Hemenway, P. D., Shelus, P. J., McArthur, B., McCartney, J. E., Franz, O. G., Wasserman, L. W., Kreidl, T. J. N., van Altena, Wm. F., Girard, T., and Fredrick, L. W., 1991, in The First Year of HST Observations, eds. A. L. Kinney and J. C. Blades, Space Telescope Science Institute, Baltimore, MD, pg. 131.

Bradley, A., Abramowicz-Reed, L., Story, D., Benedict, G., and Jefferys, W. 1991, PASP 103, 317.

Burrows, C. J., Holtzman, J. A., Faber, S. M., Bely, P. Y., Hasan, H., Lynds, C. R., and Schroeder, D. 1991, ApJ 369, L21.

Franz, O. G., Kreidl, T. J. N., Wasserman, L. W., Bradley, A. J., Benedict, G. F., Hemenway, P. D., Jefferys, W. H., McArthur, B., McCartney, J. E., Nelan, E., Shelus, P. J., Story, D., Whipple, A. L., Duncombe, R. L., Fredrick, L. W., and van Altena, Wm. F. 1991, ApJ 377, L17.

Franz, O. G., Wasserman, L. H., Nelan, E., Lattanzi, M. G., Bucciarelli, B., and Taff, L. G. 1992, AJ 103, 190.

Green, R. M. 1985, Spherical Astronomy, Camb. Univ. Press.

Kamper, K. W. and Wesselink, A. J. 1978, AJ 83, 1653.

Lattanzi, M. G., Bucciarelli, B., Holfeltz, S. T., and Taff, L. G. 1992, in the proceedings of IAU Colloq. No. 135.

Porter, J. G. and Sadler, D. H. 1953, MNRAS 113, 455.

Sandage, A. R. 1962, ApJ 135, 333.

Schwarzschild, K., 1894, Astron. Nachr. 136, 81.

Scott, F. P. and Hughes, J. A. 1964, AJ 369, 368.

Stumpff, P., 1985, A\&A 144, 232.

Taff, L. G., 1990, ApJ 365, 407. . 1991, Adv. Space Res. 11, 97.

.1992a, Hubble Space Telescope Fine Guidance Sensor Instrument Handbook, Version 3.0, ST ScI, Baltimore, MD. .1992b, submitted to ApJ.

Taff, L. G., Lattanzi, M. G., Bucciarelli, B., Gilmozzi, R., McClean, B. J., Jenkner, M., Laidler, V. G., Lasker, B. M., Shara, M. M., and Sturch, C. R., 1990, ApJ 353, L45.

Upgren, A. R., Mesrobian, W. S., and Kerridge, S. J. 1972, AJ 77, 74.

Walter, H. G., 1985, in Proceedings of the Second F.A.S.T. Thinkshop, CERGA, Grasse, p. 323.

Walter, H. G., Mignard, F., Hering, R., Froeschle, M., and Falin, J. L., 1986, Manu. Geod. $11,103$. 